\title{
HYPERUNIFORM POINT SETS ON THE SPHERE: PROBABILISTIC ASPECTS
}

\author{
JOHANN S. BRAUCHART ${ }^{\dagger}$, PETER J. GRABNER ${ }^{\ddagger}$, WÖDEN KUSNER ${ }^{\ddagger}$, \\ AND JONAS ZIEFLE
}

\begin{abstract}
The concept of hyperuniformity has been introduced by Torquato and Stillinger in 2003 as a notion to detect structural behaviour intermediate between crystalline order and amorphous disorder. The present paper studies a generalisation of this concept to the unit sphere. It is shown that several well studied determinantal point processes are hyperuniform.
\end{abstract}

\section{INTRODUCTION}

It has been observed for a long time in the physics literature that large (ideally infinite) particle systems can exhibit structural behaviour between crystalline order and total disorder. Very prominent examples are given by quasi-crystals and jammed sphere packings. Research in mathematics and physics has been inspired by the discovery of such materials which lie between crystalline order and amorphous disorder. We just mention de Bruijn's Fourier analytic explanation for the diffraction pattern of quasi-crystals [8] and the extensive collection of articles on quasi-crystals [4 as examples.

Hyperuniformity was introduced in [22] as a concept to measure the occurrence of "intermediate" order. Such hyperuniform configurations $X$ occur in jammed packings, in colloids, as well as in quasi-crystals. The main feature of hyperuniformity is the fact that local density fluctuations ("number variance") are of smaller order than for an i.i.d. random ("Poissonian") point configuration.

The point of view taken in 22] was probabilistic based on point processes. It has since been observed that determinantal point processes

2010 Mathematics Subject Classification. 60G55 (Primary) 11K38 65C05 82D30 (Secondary).

$\dagger$ This author is supported by the Lise Meitner scholarship M 2030 of the Austrian Science Foundation FWF.

$\ddagger$ These authors were supported by the Austrian Science Fund FWF project F5503 (part of the Special Research Program (SFB) "Quasi-Monte Carlo Methods: Theory and Applications"). 
exhibit less disordered behaviour in comparison to i.i.d. points due to the built in mutual repulsion of particles (see [10]). The prototypical example of such a point process is given by the distribution of fermionic particles, whose joint wave function is given as a determinant expressed in terms of the individual wave functions.

An infinite discrete point set $X \subset \mathbb{R}^{d}$ is defined to be hyperuniform if the variance of the random variable ("number variance") \# $((\mathbf{x}+$ $t \Omega) \cap X$ ) behaves like $o\left(t^{d}\right)$ as $t \rightarrow \infty$. Here, $\Omega$ is a fixed compact test set ("window"); in most of the cases $\Omega$ is chosen as a Euclidean ball. Notice that the number variance for i.i.d. point sets is of exact order $t^{d}$. Thus, hyperuniformity is characterised by a smaller order of magnitude of the variance. It was shown in 22 that the best possible order for the variance is $t^{d-1}$.

In [7] a notion of hyperuniformity for sequences of finite point sets on the sphere was introduced. In that paper three regimes of hyperuniformity were identified and studied, and several sequences of deterministically given point sets such as designs, QMC-designs, and certain energy minimising point sets were shown to exhibit hyperuniform behaviour. We also refer the reader to related recent work [15,17].

It is the aim of the present paper to study hyperuniformity on the sphere for samples of point processes on the sphere. Especially, we study the spherical ensemble (see [10,11]) on $\mathbb{S}^{2}$ (Section [5]), the harmonic ensemble introduced in [5] (Section 6), and the jittered sampling process (Section 7). We observe that the jittered sampling process can be seen as a determinantal point process. All processes turn out to be hyperuniform in all three regimes. The harmonic ensemble has slightly weaker behaviour in the threshold order regime.

Throughout this paper $\sigma=\sigma_{d}$ will denote the normalised surface area measure on $\mathbb{S}^{d}$. We suppress the dependence on $d$ in this notation.

\section{Point Processes}

We consider a point process $\mathscr{X}_{N}$ sampling $N$ points given by the joint densities $\rho^{(N)}$ in the sense that

$$
\mathbb{P}\left(\left(X_{1}, \ldots, X_{N}\right) \in B\right)=\int \cdots \int_{B} \rho^{(N)}\left(\mathbf{x}_{1}, \ldots, \mathbf{x}_{N}\right) \mathrm{d} \sigma\left(\mathbf{x}_{1}\right) \cdots \mathrm{d} \sigma\left(\mathbf{x}_{N}\right),
$$

where $B$ is a measurable subset of $\left(\mathbb{S}^{d}\right)^{N}$. We will assume throughout this paper that the number of points $N$ is fixed and that the process is simple, which means that the probability of sampling a point more than once is zero. In some of the studied examples the number of points will depend on a parameter $L$; in these cases we write $N_{L}$ for this number. 
Note that in the literature (e.g., [10]) the process is often given in terms of its joint intensities (correlation functions) which are given by $N ! \cdot \rho^{(N)}$. We use joint densities with respect to the natural measure $\sigma$ on $\mathbb{S}^{d}$ in this paper since they make the asymptotic dependence on $N$ more transparent. By a result of Lenard [20], locally integrable functions $\rho^{(N)}$ can be represented as the joint densities of a point process if and only if they satisfy a positivity condition and the particles are exchangeable; i.e., the joint densities are invariant under permutation of the entries

$$
\rho^{(N)}\left(\mathbf{x}_{\tau(1)}, \ldots, \mathbf{x}_{\tau(N)}\right)=\rho^{(N)}\left(\mathbf{x}_{1}, \ldots, \mathbf{x}_{N}\right) \text { for all } \mathbf{x}_{i} \in \mathbb{S}^{d}, \tau \in \mathrm{S}_{N} .
$$

The reduced densities

$$
\rho_{k}^{(N)}\left(\mathbf{x}_{1}, \ldots, \mathbf{x}_{k}\right):=\int_{\left(\mathbb{S}^{d}\right)^{N-k}} \rho^{(N)}\left(\mathbf{x}_{1}, \ldots, \mathbf{x}_{N}\right) \mathrm{d} \sigma\left(\mathbf{x}_{k+1}\right) \cdots \mathrm{d} \sigma\left(\mathbf{x}_{N}\right),
$$

$1 \leq k \leq N$, describe how $k$ of $N$ points are distributed. The joint intensities are $\frac{N !}{(N-k) !} \rho_{k}^{(N)}$.

The number of points that are put into a test set $B \subseteq \mathbb{S}^{d}$ by the process is the random variable $\mathscr{X}_{N}(B):=\sum_{i=1}^{N} \mathbb{1}_{B}\left(X_{i}\right)$, or in other words $N$ times the empirical measure of $B$. As usual, $\mathbb{1}_{B}$ denotes the indicator function of the set $B$.

For most of our study, we restrict ourselves to processes that are invariant under isometries of the sphere

$$
\begin{aligned}
\rho^{(N)}\left(A \mathbf{x}_{1}, \ldots, A \mathbf{x}_{N}\right)= & \rho^{(N)}\left(\mathbf{x}_{1}, \ldots, \mathbf{x}_{N}\right) \\
& \text { for all } \mathbf{x}_{i} \in \mathbb{S}^{d}, A \in \mathrm{SO}(d+1) .
\end{aligned}
$$

By summation over permutations and integration over isometries, joint densities satisfying (11) and (2) do exist. In this case we obtain

$$
\begin{aligned}
\mathbb{E} \mathscr{X}_{N}(B)= & N \sigma(B) \\
\mathbb{V} \mathscr{X}_{N}(B)= & \mathbb{E}\left(\mathscr{X}_{N}(B)^{2}\right)-\left(\mathbb{E} \mathscr{X}_{N}(B)\right)^{2} \\
= & N \sigma(B)(1-\sigma(B)) \\
& +N(N-1) \iint_{B \times B}\left(\rho_{2}^{(N)}\left(\mathbf{x}_{1}, \mathbf{x}_{2}\right)-1\right) \mathrm{d} \sigma\left(\mathbf{x}_{1}\right) \mathrm{d} \sigma\left(\mathbf{x}_{2}\right) .
\end{aligned}
$$

The variance is independent of the position and orientation of the test set $B$. So for a spherical cap the number variance only depends on the radius of the cap.

Determinantal Point Processes. Following [10, we introduce determinantal point processes on $\mathbb{S}^{d}$. As pointed out before, we formulate the description in terms of joint densities, rather than joint intensities. 
Definition 1. A simple point process on $\mathbb{S}^{d}$ is called determinantal with kernel $K$ if its joint densities with respect to $\sigma$ are given by

$$
\rho_{k}^{(N)}\left(\mathbf{x}_{1}, \ldots, \mathbf{x}_{k}\right)=\frac{(N-k) !}{N !} \operatorname{det}\left(K\left(\mathbf{x}_{i}, \mathbf{x}_{j}\right)\right)_{i, j=1}^{k}, \quad 1 \leq k \leq N .
$$

From the definition, permutations of the variables do not change the process. Furthermore, if $\mathbf{x}_{i}=\mathbf{x}_{j}$ for some $i \neq j$, then the density is zero.

In [10] it is shown that a process $\mathscr{X}_{N}$ samples exactly $N$ points if and only if it is associated with the projection of $L^{2}$ to an $N$-dimensional subspace $H$. Let $\psi_{1}, \ldots, \psi_{N}$ be an orthonormal basis of $H$, then the kernel is given by

$$
K_{H}(\mathbf{x}, \mathbf{y})=\sum_{i=1}^{N} \psi_{i}(\mathbf{x}) \overline{\psi_{i}(\mathbf{y})}
$$

\section{Hyperuniformity on the Sphere}

Complementing the extensive study of the notion of hyperuniformity in the infinite setting, we are interested in studying an analogous property of sequences of point sets in compact spaces. For convenience, we study the $d$-dimensional unit sphere $\mathbb{S}^{d}$. Our ideas immediately generalise to homogeneous spaces; further generalisations might be more elaborate, since we rely heavily on harmonic analysis and specific properties of special functions. For instance, for the flat torus a similar study has been carried out in [21].

In order to adapt to the compact setting, we replace the infinite set $X$ studied in the classical notion of hyperuniformity by a sequence of finite point sets, $\left(X_{N}\right)_{N \in \mathcal{J}}$, where we assume that the cardinality $\# X_{N}$ is $N$. By using an infinite set $\mathcal{J} \subseteq \mathbb{N}$ as index set, we always allow for subsequences.

Throughout the paper we use the notation

$$
C(\mathbf{x}, \phi)=\left\{\mathbf{y} \in \mathbb{S}^{d} \mid\langle\mathbf{x}, \mathbf{y}\rangle>\cos \phi\right\}
$$

for the (open) spherical cap with center $\mathbf{x}$ and opening angle $\phi$. The normalised surface area of the cap is given by

$$
\sigma(C(\mathbf{x}, \phi))=\gamma_{d} \int_{0}^{\phi} \sin (\theta)^{d-1} \mathrm{~d} \theta \asymp \phi^{d} \quad \text { as } \phi \rightarrow 0,
$$

where

$$
\gamma_{d}=\left(\int_{0}^{\pi} \sin (\theta)^{d-1} \mathrm{~d} \theta\right)^{-1}=\frac{\Gamma(d)}{2^{d-1} \Gamma(d / 2)^{2}} .
$$


Notice that $\gamma_{d}=\frac{\omega_{d-1}}{\omega_{d}}$, where $\omega_{d}$ is the surface area of $\mathbb{S}^{d}$. Here and throughout the paper, we shall use $f(x) \asymp g(x)$ as $x \rightarrow x_{0}$ to mean that there exist positive constants $c$ and $C$ such that $c g(x) \leq f(x) \leq C g(x)$ for $x$ sufficiently close to $x_{0}$. We will write $\sigma(C(\phi))$ for the normalised surface area of the cap $C(\cdot, \phi)$.

In this paper we shall study the number variance.

Definition 2 (Number variance). Let $\mathscr{X}_{N}$ be a point process on the sphere $\mathbb{S}^{d}$ sampling $N$ points. The number variance of $\mathscr{X}_{N}$ for caps of opening angle $\phi$ is given by

$$
V\left(\mathscr{X}_{N}, \phi\right):=\mathbb{V} \mathscr{X}_{N}(C(\cdot, \phi)):=\mathbb{E}\left(\mathscr{X}_{N}(C(\cdot, \phi))^{2}\right)-\left(\mathbb{E} \mathscr{X}_{N}(C(\cdot, \phi))\right)^{2} .
$$

If the process $\mathscr{X}_{N}$ is rotation invariant, the implicit integration with respect to the center of the cap $C(\cdot, \phi)$ can be omitted.

As in the Euclidean case we define hyperuniformity by a comparison between the behaviour of the number variance of a sequence of point sets and of the i.i.d. case. For i.i.d. random points, the variance is $N \sigma(C(\phi))(1-\sigma(C(\phi)))$ (see (4)), which has order of magnitude $N$, $N \sigma\left(C\left(\phi_{N}\right)\right)$, and $t^{d}$, respectively, in the three cases (91), (10), and (11) listed below.

Definition 3 (Hyperuniformity). Let $\mathscr{X}_{N}$ be a point process on the sphere $\mathbb{S}^{d}$ sampling $N$ points. The process $\left(\mathscr{X}_{N}\right)$ is called

- hyperuniform for large caps if

$$
V\left(\mathscr{X}_{N}, \phi\right)=o(N) \quad \text { as } N \rightarrow \infty
$$

for all $\phi \in\left(0, \frac{\pi}{2}\right)$;

- hyperuniform for small caps if

$$
V\left(\mathscr{X}_{N}, \phi_{N}\right)=o\left(N \sigma\left(C\left(\phi_{N}\right)\right)\right) \quad \text { as } N \rightarrow \infty
$$

and all sequences $\left(\phi_{N}\right)_{N \in \mathbb{N}}$ such that

(1) $\lim _{N \rightarrow \infty} \phi_{N}=0$

(2) $\lim _{N \rightarrow \infty} N \sigma\left(C\left(\phi_{N}\right)\right)=\infty$, which is equivalent to $\phi_{N} N^{\frac{1}{d}} \rightarrow \infty$ as $N \rightarrow \infty$.

- hyperuniform for caps at threshold order if

$$
\limsup _{N \rightarrow \infty} V\left(\mathscr{X}_{N}, t N^{-\frac{1}{d}}\right)=\mathcal{O}\left(t^{d-1}\right) \quad \text { as } t \rightarrow \infty .
$$

The $\mathcal{O}\left(t^{d-1}\right)$ in (11) could be replaced by the less strict $o\left(t^{d}\right)$ in a more general setting. 


\section{Intersection Volume of Spherical Caps}

In this section we collect some formulas and properties of the intersection volume of two spherical caps that will be needed in the discussion later on. Besides a possibly new formula for the volume of the intersection of two caps of equal size we provide sharp inequalities and asymptotic expansions, which enable us to obtain precise results on the number variance.

We will briefly introduce some basic facts and notation regarding spherical harmonics. Let $\mathcal{H}_{\ell}$ denote the vector space of spherical harmonics of degree $\ell \in \mathbb{N}$. Its dimension is

$$
Z(d, \ell)=\frac{2 \ell+d-1}{d-1}\left(\begin{array}{c}
\ell+d-2 \\
d-2
\end{array}\right) .
$$

With respect to the $L^{2}\left(\mathbb{S}^{d}, \sigma\right)$ inner product, $\mathcal{H}_{\ell}$ has a real orthonormal basis $\left\{Y_{\ell, k}\right\}_{k=1}^{Z(d, \ell)}$. The addition theorem for spherical harmonics (see [19]) gives

$$
\sum_{k=1}^{Z(d, \ell)} Y_{\ell, k}(\mathbf{x}) Y_{\ell, k}(\mathbf{y})=Z(d, \ell) P_{\ell}^{(d)}(\langle\mathbf{x}, \mathbf{y}\rangle), \quad \mathbf{x}, \mathbf{y} \in \mathbb{S}^{d},
$$

where $P_{\ell}^{(d)}, \ell \geq 0$, are the Legendre polynomials for the sphere $\mathbb{S}^{d}$ normalised by $P_{\ell}^{(d)}(1)=1$. Notice that for $d \geq 2$ these are Gegenbauer polynomials for the parameter $\frac{d-1}{2}$ :

$$
Z(d, \ell) P_{\ell}^{(d)}(x)=\frac{2 \ell+d-1}{d-1} C_{\ell}^{\frac{d-1}{2}}(x) .
$$

It is well-known that the Laplace series for the indicator function of the spherical cap $C(\mathbf{x}, \phi)$ is given by

$$
\mathbb{1}_{C(\mathbf{x}, \phi)}(\mathbf{y})=\sigma(C(\cdot, \phi))+\sum_{n=1}^{\infty} a_{n}(\phi) Z(d, n) P_{n}^{(d)}(\langle\mathbf{x}, \mathbf{y}\rangle),
$$

where the Laplace coefficients are given by

$$
a_{n}(\phi)=\gamma_{d} \int_{0}^{\phi} P_{n}^{(d)}(\cos (\theta)) \sin (\theta)^{d-1} \mathrm{~d} \theta=\frac{\gamma_{d}}{d} \sin (\phi)^{d} P_{n-1}^{(d+2)}(\cos (\phi))
$$

for $n \geq 1$. The intersection volume is then obtained as the spherical convolution of the indicator function with itself. This gives

$$
\begin{aligned}
g_{\phi}(\langle\mathbf{x}, \mathbf{y}\rangle) & :=\sigma(C(\mathbf{x}, \phi) \cap C(\mathbf{y}, \phi))-\sigma(C(\phi))^{2} \\
& =\sum_{n=1}^{\infty} a_{n}(\phi)^{2} Z(d, n) P_{n}^{(d)}(\langle\mathbf{x}, \mathbf{y}\rangle) .
\end{aligned}
$$


In 13. formulas for the volume of the intersection of two spherical caps have been derived. In our special case of the intersection of two caps of equal size, we get

$\sigma(C(\mathbf{x}, \phi) \cap C(\mathbf{y}, \phi))=\frac{d-1}{\pi} \int_{\frac{\psi}{2}}^{\phi} \sin (t)^{d-1} \int_{0}^{\arccos \left(\frac{\tan \left(\frac{\psi}{2}\right)}{\tan (t)}\right)} \sin (u)^{d-2} \mathrm{~d} u \mathrm{~d} t$,

where $\langle\mathbf{x}, \mathbf{y}\rangle=\cos \psi$ and $\psi \leq 2 \phi$.

The change of variables

$$
\begin{aligned}
& \tan (v)=\tan (t) \cos (u), \\
& \sin (w)=\sin (t) \sin (u)
\end{aligned}
$$

transforms the double integral into

$$
\frac{1}{\pi} \int_{\frac{\psi}{2}}^{\phi} \frac{\left(\sin ^{2} \phi-\sin ^{2} v\right)^{\frac{d-1}{2}}}{\cos (v)^{d-1}} \mathrm{~d} v
$$

This gives

$g_{\phi}(1)-g_{\phi}(\cos \psi)=\sigma(C(\mathbf{x}, \phi) \backslash C(\mathbf{y}, \phi))=\frac{1}{\pi} \int_{0}^{\frac{\psi}{2}} \frac{\left(\sin ^{2} \phi-\sin ^{2} v\right)_{+}^{\frac{d-1}{2}}}{\cos (v)^{d-1}} \mathrm{~d} v$

for all $0<\psi<\pi$; here we define $(a)_{+}:=\max (0, a)$.

From this we obtain the following lemma.

Lemma 1. There exists a positive constant $A_{d}$ depending only on $d$ such that for all $(\phi, \psi)$ with $0 \leq \psi \leq 2 \phi \leq \pi$ the inequalities

$\frac{1}{2 \pi} \psi(\sin \phi)^{d-1}-A_{d} \psi^{3} \sin (\phi)^{d-3} \leq \sigma(C(\mathbf{x}, \phi) \backslash C(\mathbf{y}, \phi)) \leq \frac{1}{2 \pi} \psi(\sin \phi)^{d-1}$

hold. Here, $\cos \psi=\langle\mathbf{x}, \mathbf{y}\rangle$. For $d \leq 3$, these inequalities hold for $(\phi, \psi) \in\left[0, \frac{\pi}{2}\right] \times[0, \pi]$.

Proof. In the integral (15) we make the substitution $\sin (v)=\sin (\phi) \sin (u)$ to obtain

$$
g_{\phi}(1)-g_{\phi}(\cos \psi)=\frac{\sin (\phi)^{d}}{\pi} \int_{0}^{h(\phi, \psi)} \frac{\cos (u)^{d}}{\left(1-\sin (\phi)^{2} \sin (u)^{2}\right)^{\frac{d}{2}}} \mathrm{~d} u,
$$

where the upper bound in integration is given by

$$
h(\phi, \psi)=\arcsin \left(\min \left(1, \frac{\sin \left(\frac{\psi}{2}\right)}{\sin (\phi)}\right)\right) .
$$


Now, the integrand in (17) is bounded from below by $\cos (u)^{d} \geq 1-\frac{d}{2} u^{2}$, from which we derive the estimate

$g_{\phi}(1)-g_{\phi}(\cos \psi) \geq \frac{\sin (\phi)^{d}}{\pi}\left(\arcsin \left(\frac{\sin \left(\frac{\psi}{2}\right)}{\sin (\phi)}\right)-\frac{d}{6}\left(\arcsin \left(\frac{\sin \left(\frac{\psi}{2}\right)}{\sin (\phi)}\right)\right)^{3}\right)$.

From this we derive the lower bound in (16) using the estimates

$$
\begin{aligned}
x & \leq \arcsin (x) \leq x+\left(\frac{\pi}{2}-1\right) x^{3} \\
x-\frac{x^{3}}{3} & \leq \sin (x) \leq x
\end{aligned}
$$

for $x \geq 0$.

For the upper bound, we just observe that the integrand in (15) is bounded by $\sin (\phi)^{d-1}$.

For $d=2$, we get the explicit formula

$$
\begin{aligned}
& \sigma(C(\mathbf{x}, \phi) \backslash C(\mathbf{y}, \phi)) \\
& = \begin{cases}\frac{1}{\pi}\left(\arcsin \left(\frac{\sin \frac{\psi}{2}}{\sin \phi}\right)-\arcsin \left(\frac{\tan \frac{\psi}{2}}{\tan \phi}\right) \cos \phi\right) & \text { for } \psi \leq 2 \phi \\
\sin ^{2} \frac{\phi}{2} & \text { for } \psi>2 \phi,\end{cases}
\end{aligned}
$$

where $\cos \psi=\langle\mathbf{x}, \mathbf{y}\rangle$.

\section{The Spherical Ensemble}

The spherical ensemble of $N$ points is obtained by stereographically projecting the eigenvalues of $A^{-1} B$ to the sphere $\mathbb{S}^{2}$, where $A$ and $B$ are $N \times N$ matrices with i.i.d. random complex Gaussian entries (see [10, Section 4.3.8] or [11, Chapter 5]).

These eigenvalues form a determinantal point process $\mathscr{X}_{N}^{S}$ on $\mathbb{C}$ with kernel

$$
\widetilde{K}_{N}(z, w):=(1+z \bar{w})^{N-1}
$$

with respect to the measure

$$
\mathrm{d} \mu_{N}(z):=\frac{N}{\pi\left(1+|z|^{2}\right)^{N+1}} \mathrm{~d} \lambda_{2}(z)
$$

where $\lambda_{2}$ denotes the Lebesgue measure on $\mathbb{C}$. The corresponding function space is the space of square integrable entire functions

$$
\mathscr{P}_{N}:=L^{2}\left(\mathbb{C}, \mathrm{d} \mu_{N}\right) \cap H(\mathbb{C}),
$$

which consists exactly of the polynomials of degree $\leq N-1$. The kernel $\widetilde{K}_{N}$ is the reproducing kernel of this Hilbert space. 
Applying the stereographic projection

$$
\mathbf{x}=\left(x_{1}, x_{2}, x_{3}\right) \in \mathbb{S}^{2} \mapsto \frac{x_{1}+i x_{2}}{1-x_{3}}
$$

transforms the measure by

$$
\mathrm{d} \mu_{N}(z)=\frac{N}{2^{N-1}}\left(1-x_{3}\right)^{N-1} \mathrm{~d} \sigma(\mathbf{x}) .
$$

In order to obtain an isometry of spaces $L^{2}\left(\mathbb{C}, \mu_{N}\right)$ and $L^{2}\left(\mathbb{S}^{2}, \sigma\right)$, the basis elements of $\mathscr{P}_{N}$ are mapped by

$$
z^{k} \mapsto \frac{\sqrt{N}}{2^{\frac{N-1}{2}}}\left(x_{1}+i x_{2}\right)^{k}\left(1-x_{3}\right)^{\frac{N-1}{2}-k} \text { for } k=0, \ldots, N-1 .
$$

Inserting (19) into (18) and multiplying by $\frac{N}{2^{N-1}}\left(\left(1-x_{3}\right)\left(1-y_{3}\right)\right)^{\frac{N-1}{2}}$ gives the projected kernel on the sphere

$$
K_{N}(\mathbf{x}, \mathbf{y})=\frac{N}{2^{N-1}}\left(\frac{1+\langle\mathbf{x}, \mathbf{y}\rangle-x_{3}-y_{3}+i\left(x_{2} y_{1}-x_{1} y_{2}\right)}{\sqrt{\left(1-x_{3}\right)\left(1-y_{3}\right)}}\right)^{N-1}
$$

and the space of functions on $\mathbb{S}^{2}$ is spanned by the functions given in (21). These functions are orthogonal with respect to $\sigma$.

In order to compute the expectation of a general energy sum with respect to the process generated by $K_{N}$, we compute the determinant

$$
N(N-1) \rho_{2}^{(N)}(\mathbf{x}, \mathbf{y})=K_{N}(\mathbf{x}, \mathbf{x}) K_{N}(\mathbf{y}, \mathbf{y})-\left|K_{N}(\mathbf{x}, \mathbf{y})\right|^{2} .
$$

We notice that $K_{N}(\mathbf{x}, \mathbf{x})=N$ and

$$
|1+z \bar{w}|^{2}=\left|\left(1+\frac{x_{1}+i x_{2}}{1-x_{3}} \frac{y_{1}-i y_{2}}{1-y_{3}}\right)\right|^{2}=\frac{1}{\left(1-x_{3}\right)\left(1-y_{3}\right)}(2+2\langle\mathbf{x}, \mathbf{y}\rangle)
$$

for $\mathbf{x}, \mathbf{y} \in \mathbb{S}^{2}$, which gives

$$
\left|K_{N}(\mathbf{x}, \mathbf{y})\right|^{2}=N^{2}\left(\frac{1+\langle\mathbf{x}, \mathbf{y}\rangle}{2}\right)^{N-1}
$$

and

$$
N(N-1) \rho_{2}^{(N)}(\mathbf{x}, \mathbf{y})=N^{2}\left(1-\left(\frac{1+\langle\mathbf{x}, \mathbf{y}\rangle}{2}\right)^{N-1}\right)
$$


Now let $g:[-1,1] \rightarrow \mathbb{R}$ be a function with $\int_{-1}^{1} g(x) \mathrm{d} x=0$. Then

$$
\begin{aligned}
&(22) \quad E_{g}(N):=\mathbb{E} \sum_{i, j=1}^{N} g\left(\left\langle\mathbf{x}_{i}, \mathbf{x}_{j}\right\rangle\right) \\
&=N g(1)+N^{2} \iint_{\mathbb{S}^{2} \times \mathbb{S}^{2}} g(\langle\mathbf{x}, \mathbf{y}\rangle)\left(1-\left(\frac{1+\langle\mathbf{x}, \mathbf{y}\rangle}{2}\right)^{N-1}\right) \mathrm{d} \sigma(\mathbf{x}) \mathrm{d} \sigma(\mathbf{y}) \\
&=\frac{N^{2}}{2} \int_{-1}^{1}(g(1)-g(x))\left(\frac{1+x}{2}\right)^{N-1} \mathrm{~d} x .
\end{aligned}
$$

We apply (22) to the function $g_{\phi}$ given by (14). Putting everything together, we obtain

$$
\begin{aligned}
V\left(\mathscr{X}_{N}^{S}, \phi\right)= & E_{g_{\phi}}(N) \\
= & \frac{N^{2}}{4 \pi} \sin \phi \int_{-1}^{1} \arccos (x)\left(\frac{1+x}{2}\right)^{N-1} \mathrm{~d} x \\
& +\mathcal{O}\left(\frac{N^{2}}{\sin \phi} \int_{-1}^{1} \arccos (x)^{3}\left(\frac{1+x}{2}\right)^{N-1} \mathrm{~d} x\right) \\
= & \frac{\sin \phi}{2 \sqrt{\pi}} \frac{\Gamma\left(N+\frac{1}{2}\right)}{\Gamma(N)}+\mathcal{O}\left(\frac{1}{N^{1 / 2} \sin \phi}\right) \\
= & \frac{\sqrt{\sigma(C(\phi))(1-\sigma(C(\phi)))}}{\sqrt{\pi}} N^{1 / 2}+\mathcal{O}\left(\frac{1}{N^{1 / 2} \sin \phi}\right)
\end{aligned}
$$

valid for $\phi \in\left(0, \frac{\pi}{2}\right)$. Thus, we have proved the following lemma. We note that (24) was obtained in [2, Lemma 2.1] with the restriction that $\sigma(C(\phi))^{-1}=o(N)$ and with a weaker error term.

Lemma 2. The number variance of the spherical ensemble satisfies for $\phi \in(0, \pi)$

$$
V\left(\mathscr{X}_{N}^{S}, \phi\right)=\frac{\sqrt{\sigma(C(\phi))(1-\sigma(C(\phi)))}}{\sqrt{\pi}} N^{1 / 2}+\mathcal{O}\left(\frac{1}{N^{1 / 2} \sin \phi}\right)
$$

with an absolute implied constant; especially,

$$
\lim _{N \rightarrow \infty} V\left(\mathscr{X}_{N}^{S}, t N^{-\frac{1}{2}}\right)=\frac{t}{2 \sqrt{\pi}}+\mathcal{O}\left(t^{-1}\right)
$$

Remark 1. Inserting (15) directly into (22) gives the closed formula

$$
E_{g_{\phi}}(N)=\frac{N \sin ^{2} \phi}{\pi} \int_{0}^{1}\left(1-v^{2}\right)^{\frac{1}{2}}\left(1-v^{2} \sin ^{2} \phi\right)^{N-1} \mathrm{~d} v,
$$


which could be used for an alternative yet slightly more elaborate proof of Lemma 2,

From this lemma we immediately obtain the following theorem.

Theorem 1. The spherical ensemble is hyperuniform in all three regimes.

Proof. For the large cap case, we obtain $V\left(\mathscr{X}_{N}^{S}, \phi\right)=\mathcal{O}\left(N^{1 / 2}\right)$; for the small cap case, we obtain $V\left(\mathscr{X}_{N}^{S}, \phi_{N}\right)=\mathcal{O}\left(\left(N \phi_{N}\right)^{1 / 2}\right)=o\left(N \phi_{N}\right)$. In the threshold order case, we use (26).

Remark 2. The error term in (25) has the correct order with respect to $N$ and $\phi$. This shows that taking $\phi_{N}=o\left(N^{-\frac{1}{2}}\right)$ does not make sense, because then the error term would become the dominant term that tends to $\infty$.

\section{The Harmonic Ensemble}

The function space of spherical harmonics of degree $\leq L$ on the sphere $\mathbb{S}^{d}$ and the projection kernel to this space of dimension $Z(d+$ $1, L)=\frac{2 L+d}{d}\left(\begin{array}{c}L+d-1 \\ d-1\end{array}\right)$ was used in [5] to define a determinantal point process $\mathscr{X}_{L}^{H}$, the harmonic ensemble. This process samples $N:=N_{L}:=$ $Z(d+1, L) \asymp L^{d}$ points. We will study this process with respect to hyperuniformity in this section.

The projection kernel to this space is given by

$$
K_{L}(\langle\mathbf{x}, \mathbf{y}\rangle):=\sum_{\ell=0}^{L} Z(d, \ell) P_{\ell}^{(d)}(\langle\mathbf{x}, \mathbf{y}\rangle)=\frac{Z(d+1, L)}{\left(\begin{array}{c}
L+d / 2 \\
L
\end{array}\right)} \mathcal{P}_{L}^{\left(\frac{d}{2}, \frac{d}{2}-1\right)}(\langle\mathbf{x}, \mathbf{y}\rangle)
$$

for $\mathbf{x}, \mathbf{y} \in \mathbb{S}^{d}$, where $\mathcal{P}_{L}^{(\alpha, \beta)}, L \geq 0$, are the usual Jacobi polynomials. This identity follows from [3, Theorem 7.1.3] after rewriting the Legendre polynomials in terms of Gegenbauer and Jacobi polynomials using (12).

Theorem 2. The harmonic ensemble is hyperuniform for large and small caps. In the threshold order regime the weaker property

$$
\limsup _{L \rightarrow \infty} V\left(\mathscr{X}_{L}^{H}, t N_{L}^{-\frac{1}{d}}\right)=\mathcal{O}\left(t^{d-1} \log t\right)=o\left(t^{d}\right)
$$

holds.

Proof. The number variance $V\left(\mathscr{X}_{L}^{H}, \phi\right)$ can be expressed as (cf. similar computations that lead to (22))

$$
\gamma_{d} \int_{0}^{\pi}\left(g_{\phi}(1)-g_{\phi}(\cos \theta)\right) K_{L}(\cos \theta)^{2}(\sin \theta)^{d-1} \mathrm{~d} \theta
$$


where $g_{\phi}$ is given by (14). Using Lemma 1, we obtain

$$
\begin{aligned}
& V\left(\mathscr{X}_{L}^{H}, \phi\right) \\
= & \frac{\gamma_{d}}{\pi}\left(\frac{Z(d+1, L)}{\left(\begin{array}{c}
L+\frac{d}{2} \\
L
\end{array}\right)}\right)^{2}(2 \sin \phi)^{d-1} \int_{0}^{2 \phi}\left(\mathcal{P}_{L}^{\left(\frac{d}{2}, \frac{d}{2}-1\right)}(\cos \theta)\right)^{2}\left(\sin \frac{\theta}{2}\right)^{d}\left(\cos \frac{\theta}{2}\right)^{d-1} \mathrm{~d} \theta \\
+ & \mathcal{O}\left(L^{d}(\sin \phi)^{d-3} \int_{0}^{2 \phi}\left(\mathcal{P}_{L}^{\left(\frac{d}{2}, \frac{d}{2}-1\right)}(\cos \theta)\right)^{2}\left(\sin \frac{\theta}{2}\right)^{d+2}\left(\cos \frac{\theta}{2}\right)^{d-1} \mathrm{~d} \theta\right) \\
+ & \gamma_{d}\left(\frac{Z(d+1, L)}{\left(\begin{array}{c}
L+\frac{d}{2} \\
L
\end{array}\right)}\right)^{2} \sigma(C(\phi)) \int_{2 \phi}^{\pi}\left(\mathcal{P}_{L}^{\left(\frac{d}{2}, \frac{d}{2}-1\right)}(\cos \theta)\right)^{2}(\sin \theta)^{d-1} \mathrm{~d} \theta .
\end{aligned}
$$

The case of large and small caps was studied in [5]; we summarise the computations given there for completeness. The case of caps at threshold order is new and will be given in more detail. We make use of known asymptotic expansions for the Jacobi polynomials (see [16, 5.2.3 and 5.2.4])

$$
\begin{aligned}
\mathcal{P}_{L}^{\left(\frac{d}{2}, \frac{d}{2}-1\right)}(\cos \theta) & =\frac{\cos \left(\left(L+\frac{d}{2}\right) \theta-\frac{\pi}{4}(d+1)\right)}{\sqrt{\pi L}\left(\sin \frac{\theta}{2}\right)^{\frac{d+1}{2}}\left(\cos \frac{\theta}{2}\right)^{\frac{d-1}{2}}}+\mathcal{O}\left(L^{-\frac{3}{2}}\right) \\
\mathcal{P}_{L}^{\left(\frac{d}{2}, \frac{d}{2}-1\right)}\left(\cos \frac{\tau}{L}\right) & =L^{\frac{d}{2}}\left(\frac{2}{\tau}\right)^{\frac{d}{2}} J_{\frac{d}{2}}(\tau)+\mathcal{O}\left(L^{\frac{d}{2}-1}\right),
\end{aligned}
$$

where $J_{\frac{d}{2}}$ denotes the Bessel function of the first kind of index $\frac{d}{2}$. Given a constant $C>0$, the asymptotic relation (29) is used for $\theta>\frac{C}{L}$, whereas relation (30) is used for $\theta=\frac{\tau}{L} \leq \frac{C}{L}$.

This gives

$$
\begin{aligned}
\int_{0}^{\frac{C}{L}}\left(\mathcal{P}_{L}^{\left(\frac{d}{2}, \frac{d}{2}-1\right)}(\cos \theta)\right)^{2}\left(\sin \frac{\theta}{2}\right)^{d} & \left(\cos \frac{\theta}{2}\right)^{d-1} \mathrm{~d} \theta \\
& =\frac{1}{L} \int_{0}^{C} J_{\frac{d}{2}}(\theta)^{2} \mathrm{~d} \theta+\mathcal{O}\left(L^{-2}\right)
\end{aligned}
$$

for the integral over the "small" values of $\theta$,

$$
\begin{array}{r}
\int_{\frac{C}{L}}^{\alpha}\left(\mathcal{P}_{L}^{\left(\frac{d}{2}, \frac{d}{2}-1\right)}(\cos \theta)\right)^{2}\left(\sin \frac{\theta}{2}\right)^{d}\left(\cos \frac{\theta}{2}\right)^{d-1} \mathrm{~d} \theta \\
=\frac{1}{\pi L} \int_{\frac{C}{L}}^{\alpha} \frac{\cos \left(\left(L+\frac{d}{2}\right) \theta-\frac{\pi}{4}(d+1)\right)^{2}}{\sin \left(\frac{\theta}{2}\right)} \mathrm{d} \theta+\mathcal{O}\left(L^{-2}\right)
\end{array}
$$


for the integral over the "large" values of $\theta$,

$$
\begin{aligned}
& \int_{\alpha}^{\pi}\left(\mathcal{P}_{L}^{\left(\frac{d}{2}, \frac{d}{2}-1\right)}(\cos \theta)\right)^{2}\left(\sin \frac{\theta}{2}\right)^{d-1}\left(\cos \frac{\theta}{2}\right)^{d-1} \mathrm{~d} \theta \\
= & \frac{1}{\pi L} \int_{\alpha}^{\pi} \frac{\cos \left(\left(L+\frac{d}{2}\right) \theta-\frac{\pi}{4}(d+1)\right)^{2}}{\left(\sin \frac{\theta}{2}\right)^{2}} \mathrm{~d} \theta+\mathcal{O}\left(L^{-2}\right)=\mathcal{O}\left((L \alpha)^{-1}\right)
\end{aligned}
$$

and

$$
\begin{aligned}
& \text { (34) } \quad \int_{0}^{\alpha}\left(\mathcal{P}_{L}^{\left(\frac{d}{2}, \frac{d}{2}-1\right)}(\cos \theta)\right)^{2}\left(\sin \frac{\theta}{2}\right)^{d+2}\left(\cos \frac{\theta}{2}\right)^{d-1} \mathrm{~d} t \\
& =\frac{1}{\pi L} \int_{0}^{\alpha} \cos \left(\left(L+\frac{d}{2}\right) \theta-\frac{\pi}{4}(d+1)\right)^{2} \sin \left(\frac{\theta}{2}\right) \mathrm{d} \theta+\mathcal{O}\left(L^{-2}\right)=\mathcal{O}\left(\alpha^{2} L^{-1}\right)
\end{aligned}
$$

for the integral in the error term.

In the case of large caps $\left(0<\phi<\frac{\pi}{2}\right.$ fixed), we compute the number variance as

$$
\begin{array}{r}
V\left(\mathscr{X}_{L}^{H}, \phi\right)=\frac{\gamma_{d}}{\pi}\left(\frac{Z(d+1, L)}{\left(\begin{array}{c}
L+\frac{d}{2} \\
L
\end{array}\right)}\right)^{2} \frac{(2 \sin \phi)^{d-1}}{L}\left(\int_{0}^{C} J_{\frac{d}{2}}(\theta)^{2} \mathrm{~d} \theta\right. \\
\left.+\frac{1}{\pi} \int_{\frac{C}{L}}^{2 \phi} \frac{\cos \left(\left(L+\frac{d}{2}\right) \theta-\frac{\pi}{4}(d+1)\right)^{2}}{\sin \left(\frac{\theta}{2}\right)} \mathrm{d} \theta+\mathcal{O}\left(L^{-1}\right)+\mathcal{O}\left(\phi^{-1}\right)\right) \\
=\mathcal{O}\left((\sin \phi)^{d-1} L^{d-1} \log (L \phi)\right),
\end{array}
$$

where we have used $\left(Z(d+1, L) /\left(\begin{array}{c}L+\frac{d}{2} \\ L\end{array}\right)\right)^{2} \asymp L^{d}$ and the logarithmic term comes from the second summand. This is the true asymptotic order and due to $N_{L} \asymp L^{d}$ we have $V\left(\mathscr{X}_{L}^{H}, \phi\right)=o\left(N_{L}\right)$ as $L \rightarrow \infty$ for all $\phi \in\left(0, \frac{\pi}{2}\right)$.

In the case of small caps, a similar computation gives

$$
\begin{aligned}
V\left(\mathscr{X}_{L}^{H}, \phi_{L}\right) & =\mathcal{O}\left(\left(\sin \phi_{L}\right)^{d-1} L^{d-1} \log \left(L \phi_{L}\right)\right)=\mathcal{O}\left(\frac{\log \left(L \phi_{L}\right)}{L \phi_{L}}\left(\sin \phi_{L}\right)^{d} L^{d}\right) \\
& =\mathcal{O}\left(\frac{\log \left(L \phi_{L}\right)}{L \phi_{L}} N_{L} \sigma\left(C\left(\phi_{L}\right)\right)\right)=o\left(N_{L} \sigma\left(C\left(\phi_{L}\right)\right)\right) .
\end{aligned}
$$

For caps at threshold order, we analyse the three terms in (28) separately. The first term gives

$$
\frac{\gamma_{d}}{\pi}\left(\frac{Z(d+1, L)}{\left(\begin{array}{c}
L+\frac{d}{2} \\
L
\end{array}\right)}\right)^{2} \frac{\left(2 \sin t L^{-1}\right)^{d-1}}{L}\left(\int_{0}^{t} J_{\frac{d}{2}}(\theta)^{2} \mathrm{~d} \theta+\mathcal{O}\left(L^{-1}\right)\right)
$$


using (30). We use the asymptotic behaviour of the Bessel function for $\theta \rightarrow \infty($ see $[16,3.14 .1])$

$$
J_{\frac{d}{2}}(\theta)=\frac{\cos \left(\theta-\frac{\pi(d+1)}{4}\right)}{\sqrt{\frac{\pi \theta}{2}}}+\mathcal{O}\left(\theta^{-\frac{3}{2}}\right)
$$

to obtain

$$
\int_{0}^{t} J_{\frac{d}{2}}(\theta)^{2} \mathrm{~d} \theta=\frac{1}{\pi} \log t+\mathcal{O}(1)
$$

Thus the first term in (28) for $\phi_{L} \sim t L^{-1}$ is of order $t^{d-1} \log t$.

The second term in (28) is analysed using (34), which gives an order of $t^{d-1}$ for $\phi_{L} \sim t L^{-1}$. Similarly, the third term is analysed using (33), which again gives an order of $t^{d-1}$.

Putting these order estimates together yields

$$
V\left(\mathscr{X}_{L}^{H}, t L^{-1}\right)=\mathcal{O}\left(t^{d-1} \log t\right)
$$

and concludes our proof.

\section{JitTered SAmpling}

In [9] it is shown that on arbitrary Ahlfors regular metric measure spaces there exist area-regular partitions. For the case of the sphere studied here, an area regular partition is given by $\mathcal{A}=\left\{A_{1}, \ldots, A_{N}\right\}$ with $\bigcup_{i=1}^{N} A_{i}=\mathbb{S}^{d}, \sigma\left(A_{i}\right)=\frac{1}{N}$, and $i \neq j \Rightarrow A_{i} \cap A_{j}=\emptyset$ satisfying

$$
\operatorname{diam}\left(A_{i}\right) \leq C_{d} N^{-1 / d}, \quad i=1, \ldots, N,
$$

with a constant depending only on $d$ (see also [1, 6, 12, 14, 18]).

Such partitions allow us to consider the average behaviour of jittered sampling; the point process $\mathscr{X}_{N}^{\mathcal{A}}$ constructed by sampling the sphere with the condition that each of the $N$ points lies in a distinct region of the partition.

The jittered sampling variance integral is written as:

$$
\begin{aligned}
& V\left(\mathscr{X}_{N}^{\mathcal{A}}, \phi\right) \\
= & \int_{\mathbb{S}^{d}} \int_{A_{1}} \ldots \int_{A_{N}}\left(\sum_{i=1}^{N} \mathbb{1}_{C(\mathbf{x}, \phi)}\left(\mathbf{y}_{i}\right)-N \sigma(C(\phi))\right)^{2} \mathrm{~d} \sigma_{1}\left(\mathbf{y}_{1}\right) \cdots \mathrm{d} \sigma_{N}\left(\mathbf{y}_{N}\right) \mathrm{d} \sigma(\mathbf{x}),
\end{aligned}
$$


where $\sigma_{i}(\cdot):=N \sigma\left(\cdot \cap A_{i}\right)$ is the uniform probability measure on $A_{i}$. The integral can be split into off-diagonal and diagonal terms

$$
\begin{aligned}
& V\left(\mathscr{X}_{N}^{\mathcal{A}}, \phi\right)=\sum_{\substack{i, j=1 \\
i \neq j}}^{N} \int_{A_{i}} \int_{A_{j}} \sigma\left(C\left(\mathbf{y}_{i}, \phi\right) \cap C\left(\mathbf{y}_{j}, \phi\right)\right) \mathrm{d} \sigma_{i}\left(\mathbf{y}_{i}\right) \mathrm{d} \sigma_{j}\left(\mathbf{y}_{j}\right) \\
& +N \sigma(C(\phi))-N^{2} \sigma(C(\phi))^{2} \\
= & N \sum_{i=1}^{N} \int_{A_{i}}\left(\int_{\mathbb{S}^{d}} \sigma\left(C\left(\mathbf{y}_{i}, \phi\right) \cap C(\mathbf{y}, \phi)\right) \mathrm{d} \sigma(\mathbf{y})\right. \\
& \left.-\int_{A_{i}} \sigma\left(C\left(\mathbf{y}_{i}, \phi\right) \cap C(\mathbf{y}, \phi)\right) \mathrm{d} \sigma(\mathbf{y})\right) \mathrm{d} \sigma_{i}\left(\mathbf{y}_{i}\right)+N \sigma(C(\phi))-N^{2} \sigma(C(\phi))^{2} \\
= & \left.N^{2} \int_{\mathbb{S}^{d}} \int_{\mathbb{S}^{d}} \sigma(C(\mathbf{x}, \phi) \cap C(\mathbf{y}, \phi)) \mathrm{d} \sigma(\mathbf{x}) \mathrm{d} \sigma(\mathbf{y})-\sigma(C(\phi))^{2}\right) \\
& +\sum_{i=1}^{N} \int_{A_{i}} \int_{A_{i}}\left(\sigma\left(C\left(\mathbf{x}_{i}, \phi\right)\right)-\sigma\left(C\left(\mathbf{x}_{i}, \phi\right) \cap C\left(\mathbf{y}_{i}, \phi\right)\right)\right) \mathrm{d} \sigma_{i}\left(\mathbf{x}_{i}\right) \mathrm{d} \sigma_{i}\left(\mathbf{y}_{i}\right) \\
(37) & \frac{1}{2} \sum_{i=1}^{N} \int_{A_{i}} \int_{A_{i}} \sigma\left(C\left(\mathbf{x}_{i}, \phi\right) \triangle C\left(\mathbf{y}_{i}, \phi\right)\right) \mathrm{d} \sigma_{i}\left(\mathbf{x}_{i}\right) \mathrm{d} \sigma_{i}\left(\mathbf{y}_{i}\right),
\end{aligned}
$$

where $\triangle$ denotes the symmetric difference operator of two sets. For the last equality, we have used

$$
\begin{aligned}
& \int_{\mathbb{S}^{d}} \int_{\mathbb{S}^{d}} \sigma(C(\mathbf{x}, \phi) \cap C(\mathbf{y}, \phi)) \mathrm{d} \sigma(\mathbf{x}) \mathrm{d} \sigma(\mathbf{y}) \\
& =\int_{\mathbb{S}^{d}} \int_{\mathbb{S}^{d}} \int_{\mathbb{S}^{d}} \mathbb{1}_{C(\mathbf{x}, \phi)}(\mathbf{z}) \mathbb{1}_{C(\mathbf{y}, \phi)}(\mathbf{z}) \mathrm{d} \sigma(\mathbf{z}) \mathrm{d} \sigma(\mathbf{x}) \mathrm{d} \sigma(\mathbf{y}) \\
& =\int_{\mathbb{S}^{d}} \int_{\mathbb{S}^{d}} \int_{\mathbb{S}^{d}} \mathbb{1}_{C(\mathbf{z}, \phi)}(\mathbf{x}) \mathbb{1}_{C(\mathbf{z}, \phi)}(\mathbf{y}) \mathrm{d} \sigma(\mathbf{x}) \mathrm{d} \sigma(\mathbf{y}) \mathrm{d} \sigma(\mathbf{z})=\sigma(C(\phi))^{2} .
\end{aligned}
$$

So in fact the variance of the jittered sampling process reduces to the diagonal terms. The measure of the symmetric difference can be bounded like

$$
\sigma\left(C\left(\mathbf{x}_{i}, \phi\right) \triangle C\left(\mathbf{y}_{i}, \phi\right)\right) \leq C_{d} \operatorname{surface}\left(\partial C\left(\mathbf{x}_{i}, \phi\right)\right) \arccos \left(\left\langle\mathbf{x}_{i}, \mathbf{y}_{i}\right\rangle\right),
$$


where $C_{d}$ is a constant only depending on the dimension $d$. This can be seen from Lemma 1 using the observation that $\operatorname{surface}\left(\partial C\left(\mathbf{x}_{i}, \phi\right)\right) \asymp$ $(\sin \phi)^{d-1}$.

From the diameter bounds coming from our choice of equipartition, every summand in (37) can be bounded by $\mathcal{O}\left(\phi^{d-1} N^{-\frac{1}{d}}\right)$, which gives

$$
V\left(\mathscr{X}_{N}^{\mathcal{A}}, \phi\right)=\mathcal{O}\left(\phi^{d-1} N^{\frac{d-1}{d}}\right)
$$

the implied constant depends only on the dimension and the constants in (35).

Theorem 3. The jittered sampling point process is hyperuniform in all three regimes.

Proof. From (38) it is now immediate that $V\left(\mathscr{X}_{N}^{\mathcal{A}}, \phi\right)=o(N)$ for all $\phi \in\left(0, \frac{\pi}{2}\right)$, which proves hyperuniformity for large caps.

Again from (38) we obtain

$$
V\left(X_{N}, \phi_{N}\right)=\mathcal{O}\left(\left(\phi_{N} N^{\frac{1}{d}}\right)^{d-1}\right)=o\left(\left(\phi_{N} N^{\frac{1}{d}}\right)^{d}\right)=o\left(\phi_{N}^{d} N\right)
$$

under the assumptions on $\left(\phi_{N}\right)_{N \in \mathbb{N}}$ in Definition 3 , which proves hyperuniformity for small caps.

Inserting $\phi_{N}=t N^{-\frac{1}{d}}$ into (38) yields

$$
V\left(\mathscr{X}_{N}^{\mathcal{A}}, t N^{-\frac{1}{d}}\right)=\mathcal{O}\left(t^{d-1}\right) \quad \text { as } t \rightarrow \infty,
$$

which implies hyperuniformity at threshold order.

Jittered Sampling is Determinantal. In this subsection we consider a general probability space $(\Lambda, \mathcal{B}, \mu)$ with the only requirement that for any $N \in \mathbb{N}$ there exists a partition of $\Lambda$ into $N$ disjoint measurable sets of equal measure. Consider an area-regular partition $\mathcal{A}=\left\{A_{1}, \ldots, A_{N}\right\}$ of the space $\Lambda$ into pairwise disjoint measurable sets; i.e.,

$$
\begin{aligned}
A_{i} \cap A_{j} & =\emptyset, \quad i \neq j, \\
\mu\left(\bigcup_{i=1}^{N} A_{i}\right) & =1, \\
\mu\left(A_{i}\right) & =\frac{1}{N}, \quad i=1, \ldots, N .
\end{aligned}
$$

Define the projection operator

$$
p_{\mathcal{A}}(f)(x):=\sum_{i=1}^{N} \frac{\mathbb{1}_{A_{i}}(x)}{\mu\left(A_{i}\right)} \int_{A_{i}} f(y) \mathrm{d} \mu(y)=\int_{\Lambda} K_{\mathcal{A}}(x, y) f(y) \mathrm{d} \mu(y)
$$


to the space of functions measurable with respect to the finite $\sigma$-algebra generated by $\mathcal{A}$. The kernel of this operator is given by

$$
K_{\mathcal{A}}(x, y):=\sum_{i=1}^{N} \frac{\mathbb{1}_{A_{i}}(x) \mathbb{1}_{A_{i}}(y)}{\mu\left(A_{i}\right)} .
$$

The determinantal point process $\mathscr{X}_{N}^{\mathcal{A}}$ defined by the projection kernel $K_{\mathcal{A}}$ is then equal to the jittered sampling process associated to the partition $\mathcal{A}$, which can be seen by computing

$$
\begin{aligned}
\mathbb{E} \mathscr{X}_{N}^{\mathcal{A}}\left(A_{1}\right) \cdots & \mathscr{X}_{N}^{\mathcal{A}}\left(A_{N}\right) \\
& =\int_{A_{1}} \cdots \int_{A_{N}} \operatorname{det}\left(K_{\mathcal{A}}\left(x_{i}, x_{j}\right)_{i, j=1}^{N}\right) \mathrm{d} \mu\left(x_{1}\right) \cdots \mathrm{d} \mu\left(x_{N}\right) .
\end{aligned}
$$

Expanding the determinant gives

$$
\begin{aligned}
& \mathbb{E} \mathscr{X}_{N}^{\mathcal{A}}\left(A_{1}\right) \cdots \mathscr{X}_{N}^{\mathcal{A}}\left(A_{N}\right) \\
& \quad=\sum_{\pi} \operatorname{sgn}(\pi) \int_{A_{1}} \cdots \int_{A_{N}} \prod_{i=1}^{N} K_{\mathcal{A}}\left(x_{i}, x_{\pi(i)}\right) \mathrm{d} \mu\left(x_{1}\right) \cdots \mathrm{d} \mu\left(x_{N}\right) .
\end{aligned}
$$

Now we notice that $K_{\mathcal{A}}\left(x_{i}, x_{j}\right)=0$ if $i \neq j$ and $x_{i} \in A_{i}$ and $x_{j} \in A_{j}$. Thus, the integrand in the sum vanishes for all $\pi \neq \mathrm{id}$, which gives

$$
\mathbb{E} \mathscr{X}_{N}^{\mathcal{A}}\left(A_{1}\right) \cdots \mathscr{X}_{N}^{\mathcal{A}}\left(A_{N}\right)=\prod_{i=1}^{N} \int_{A_{i}} K_{\mathcal{A}}\left(x_{i}, x_{i}\right) \mathrm{d} \mu\left(x_{i}\right)=1 .
$$

The process $\mathscr{X}_{N}^{\mathcal{A}}$ samples $N$ points almost surely by the fact that $N K_{\mathcal{A}}$ defines a projection operator (see [10]). The product of random variables $\mathscr{X}_{N}^{\mathcal{A}}\left(A_{1}\right) \cdots \mathscr{X}_{N}^{\mathcal{A}}\left(A_{N}\right)$ is either 0 or 1 and thus equal to 1 (a.s.) by (39). This implies that the process samples exactly one point per set of the partition $\mathcal{A}$. Furthermore, we have

$$
\begin{aligned}
\mathbb{E} \mathscr{X}_{N}^{\mathcal{A}}(D) & =\int_{D} K(x, x) \mathrm{d} \mu(x)=\sum_{i=1}^{N} \int_{D} \frac{\mathbb{1}_{A_{i}}(x)^{2}}{\mu\left(A_{i}\right)} \mathrm{d} \mu(x) \\
& =\sum_{i=1}^{N} \frac{\mu\left(A_{i} \cap D\right)}{\mu\left(A_{i}\right)}=N \mu(D),
\end{aligned}
$$

and, for $D \subseteq A_{i}$, this implies $\mathbb{E} \mathscr{X}_{N}^{\mathcal{A}}(D)=\mu(D) / \mu\left(A_{i}\right)$; the sample point chosen from $A_{i}$ is distributed with measure $\mu_{i}$ on $A_{i}$, where

$$
\mu_{i}(A)=N \mu\left(A_{i} \cap A\right) .
$$

Acknowledgement. This material is based upon work supported by the National Science Foundation under Grant No. DMS-1439786 while 
the first three authors were in residence at the Institute for Computational and Experimental Research in Mathematics in Providence, RI, during the Spring 2018 semester.

\section{REFERENCES}

[1] R Alexander, On the sum of distances between $n$ points on a sphere, Acta Math. Hung. 23 (1972), no. 3-4, 443-448.

[2] K. Alishahi and M. Zamani, The spherical ensemble and uniform distribution of points on the sphere, Electron. J. Probab. 20 (2015), no. 23, 27.

[3] G. E. Andrews, R. Askey, and R. Roy, Special functions, Encyclopedia of Mathematics and its Applications, vol. 71, Cambridge University Press, Cambridge, 1999.

[4] F. Axel and D. Gratias (eds.), Beyond Quasicrystals, Springer, Berlin, 1995.

[5] C. Beltrán, J. Marzo, and J. Ortega-Cerdà, Energy and discrepancy of rotationally invariant determinantal point processes in high dimensional spheres, J. Complexity 37 (2016), 76-109.

[6] J. Bourgain and J. Lindenstrauss, Distribution of points on spheres and approximation by zonotopes, Israel J. Math. 64 (1988), no. 1, 25-31.

[7] J. S. Brauchart, P. J. Grabner, and W. Kusner, Hyperuniform point sets on the sphere: deterministic aspects, Constr. Approx. 50 (2019), no. 1, 45-61.

[8] N. G. de Bruijn, Quasicrystals and their Fourier transform, Indag. Math. 48 (1986), 123-152.

[9] G. Gigante and P. Leopardi, Diameter bounded equal measure partitions of Ahlfors regular metric measure spaces, Discrete Comput. Geom. 57 (2017), no. 2, 419-430.

[10] J. Ben Hough, M. Krishnapur, Y. Peres, and B. Virág, Zeros of Gaussian analytic functions and determinantal point processes, University Lecture Series, vol. 51, American Mathematical Society, Providence, RI, 2009.

[11] M. Krishnapur, Zeros of Random Analytic Functions, Ph.D. thesis, University of California, Berkeley, 2006, arXiv:math/0607504.

[12] A. Kuijlaars and E. B. Saff, Asymptotics for minimal discrete energy on the sphere, Trans. Amer. Math. Soc. 350 (1998), no. 2, 523-538.

[13] Y. Lee and W. C. Kim, Concise Formulas for the Surface Area of the Intersection of Two Hyperspherical Caps, Tech. report, Department of Industrial \& Systems Engineering, KAIST, 2014, http://ie.kaist.ac.kr/uploads/professor/tech_file/Concise+Formulas+for+the+Surface+ Area+of+the+Intersection+of+Two+Hyperspherical+Caps.pdf.

[14] P. Leopardi, A partition of the unit sphere into regions of equal area and small diameter, Electron. Trans. Numer. Anal. 25 (2006), 309-327 (electronic).

[15] A. Lošdorfer Božič and S. Čopar, Spherical structure factor and classification of hyperuniformity on the sphere, Phys. Rev. E 99 (2019), 032601.

[16] W. Magnus, F. Oberhettinger, and R. P. Soni, Formulas and theorems for the special functions of mathematical physics, Grundlehren der mathematischen Wissenschaften, vol. 52, Springer-Verlag, 1966, Third enlarged edition.

[17] A. G. Meyra, G. J. Zarragoicoechea, A. L. Maltz, E. Lomba, and S. Torquato, Hyperuniformity on spherical surfaces, Phys. Rev. E 100 (2019), 022107. 
[18] H. Mhaskar, F. Narcowich, and J. Ward, Spherical Marcinkiewicz-Zygmund inequalities and positive quadrature, Math. Comp. 70 (2001), no. 235, 11131130.

[19] C. Müller, Spherical harmonics, Lecture Notes in Mathematics, vol. 17, Springer-Verlag, Berlin, 1966.

[20] A. Soshnikov, Determinantal random point fields, Uspekhi Mat. Nauk 55 (2000), no. 5(335), 107-160. MR 1799012

[21] T. A. Stepanyuk, Hyperuniform Point Sets on Flat Tori: Deterministic and Probabilistic Aspects, Constr. Approx. (2020), to appear, https://arxiv.org/abs/1902.02973.

[22] S. Torquato and F. H. Stillinger, Local density fluctuations, hyperuniformity, and order metrics, Phys. Rev. E 68 (2003), no. 4, 041113.

(J. B., P. G.) Institute of Analysis and Number Theory, Graz UniverSity of Technology, Kopernikusgasse 24. 8010 Graz, Austria

E-mail address: j.brauchart@tugraz.at

E-mail address: peter.grabner@tugraz.at

(W. K.) Department of Mathematics, Vanderbilt University, 1326 Stevenson Center, Nashville, TN 37240, USA

E-mail address: wkusner@gmail.com

(J. Z.) Fachbereich Mathematik, Auf Der Morgenstelle 10, 72076 TüBingen, Germany

E-mail address: jonas.ziefle@googlemail.com 